\title{
Electrical activity of crystal defects in multicrystalline Si
}

\author{
A. Moretón - M.M. Jiménez • S. \\ Dadgostar • O. Martínez • M.A. \\ González • J. Jiménez .
}

Received: date / Accepted: date

\begin{abstract}
Upgraded metallurgical-grade silicon (UMGSi) solar cells with different ranges of efficiencies are characterized by light-beam induced current (LBIC) measurements. The interaction between grain boundaries (GBs) and metallic impurities is studied for cells fabricated on wafers at different solidification heights of the ingot. One observes a tight relation between the electrical activity of the grain boundaries and the position of the wafer in the ingot, which is related to the impurity contamination. The presence of a high amount of metallic impurities activates the electrical activity of the grain boundaries. The main features of the LBIC images are discussed in relation to the presence of the metallic impurities.
\end{abstract}

Keywords Multicrystalline Si · Grain Boundaries · LBIC · Impurity Gettering

\section{Introduction}

Directionally solidified multicrystalline silicon (mc-Si) shares a large part of the photovoltaic market. The availability of low cost feedstock permits a substantial reduction of solar cell production costs. However, the presence of metallic impurities in this solar grade material reduces the carrier lifetime and the photovoltaic efficiency. The efficiency losses are associated with different mechanisms, e.g., bulk recombination, surface recombination, optical losses, series resistance losses, and shunt losses [1]. Bulk recombination in mc$\mathrm{Si}$ is mainly related to the presence of crystal defects, e.g. GBs, small angle

A. Moretón · M.M. Jiménez · S. Dadgostar · O. Martínez · M.A. González · J. Jiménez GdS-Optronlab group, Dept. Física de la Materia Condensada, Universidad de Valladolid, Paseo de Belén, 19, 47011 Valladolid Spain

Tel.: +34-983-184956

Fax: +34-983-184956

E-mail: jimenez@fmc.uva.es 
grain boundaries (SAGBs), dislocations and dislocation clusters. However, one observes differences in the electrical activity of these defects, not all of them are effective traps for the photogenerated carriers. The use of spatially resolved characterization techniques is crucial to reveal the electrical activity of GBs. Among those techniques, luminescence imaging, both photoluminescence (PLi) and electroluminescence (ELi), are frequently used for full cell mapping $[2,3]$. These images provide a fast view of the distribution of non radiative recombination centers over the cell area, however, its spatial resolution does not provide a detailed analysis of the electrical activity of the crystal defects. Light beam induced current (LBIC) permits to map the spatial distribution of the bulk recombination in solar cells with micrometric resolution. It allows to study in detail the electrical activity of the GBs, as its spatial resolution is better than the one of ELi/PLi, and lock-in thermography methods [4]. The electrical activity of GBs is a matter of controversy. It has been claimed that its activity is related to the presence of metallic impurities, as they are usually found in the highest concentration around GBs and dislocation clusters [5]. The understanding of the interaction between defects and metallic impurities is a critical issue of mc-Si technology. Metallic impurities are mainly gettered by random angle GBs (RAGBs) [6], and SAGBs, which are formed by a dense line of dislocations $[5,7]$.

We present herein a study of the electrical activity of extended defects in upgraded metallurgical-grade (UMG) mc-Si, using high spatial resolution LBIC. UMG mc-Si presents high interest for the photovoltaic industry, as it provides cell efficiencies up to $20 \%$ at lower costs [8]. Also, it provides a useful way to study the interaction between defects and metallic impurities, because of its higher metallic content as compared to purified Si feedstock used in conventional mc-Si cells. The impurity concentration presents a gradient along the growth axis due to segregation. We study the electrical activity of defects along the growth axis using cells fabricated on wafers cut at different solidification heights of the ingot, which exhibit a similar structure of grains but a different concentration of impurities because of the concentration gradient along the growth axis. It has been claimed that the recombination activity of GBs depends on the position of the wafer in the ingot, which was associated with the level of contamination [9].

\section{Experimental and Samples}

The LBIC measurements were carried out in a homemade system using an $853 \mathrm{~nm}$ laser as the excitation source, for which the penetration depth in Si is $\approx 13 \mu \mathrm{m}$ [10]. A beam-splitter divides the output beam of the laser $(10 \mathrm{~mW})$ into two beams. One of them is used to measure variations in the output power through a photodiode. The other one is directed into a trinocular microscope which focuses the laser onto the solar cell. 20x, and 50x objectives were used for the measurements. The LBIC scanning is done by moving the sample with an $x-y-z$ motorized translation stage (Prior Scientific) allowing step sizes as 
small as $1 \mu \mathrm{m}$. The spatial resolution of the LBIC images is determined by the objective and step size, under selected conditions one can reach the micrometric spatial resolution. A Si CCD camera coupled to the top ocular of the microscope is used to collect the light reflected by the sample and drive an auto-focus system. The generated local photocurrent is recorded by an electrometer (Keithley Instruments). Data acquisition and hardware control were developed in LabVIEW. For more details see [11].

The solar cells were supplied by Silicio Ferrosolar (Arteixo, Spain). The cell fabrication followed the standard technological stages, including gettering and hydrogenation. The cells were fabricated on wafers cut at different heigths of the ingot. The cell efficiencies varied from $\approx 16 \%$ for the wafers in the ingot bottom to $\approx 17.8 \%$ for the wafers in the middle of the ingot.

\section{Results and Discussion}

Fig.1 presents the LBIC image (1a) and the reflected light image (1b) of a standard cell fabricated on conventional directional solidified mc-Si. The different reflectivity regions are related to the different surface texture, which depends on the grain orientation, it reveals the grains and the GBs, while small tilt angle grain boundaries are barely revealed in the reflected image. One observes in the LBIC image that most of the GBs revealed in the reflected light images present a weak electrical activity, while intragrain defects (SAGBs and dislocation clusters) appear electrically active, even if they were not revealed in the reflected light image. The LBIC image is corrected in order to compensate for the reflectivity losses.
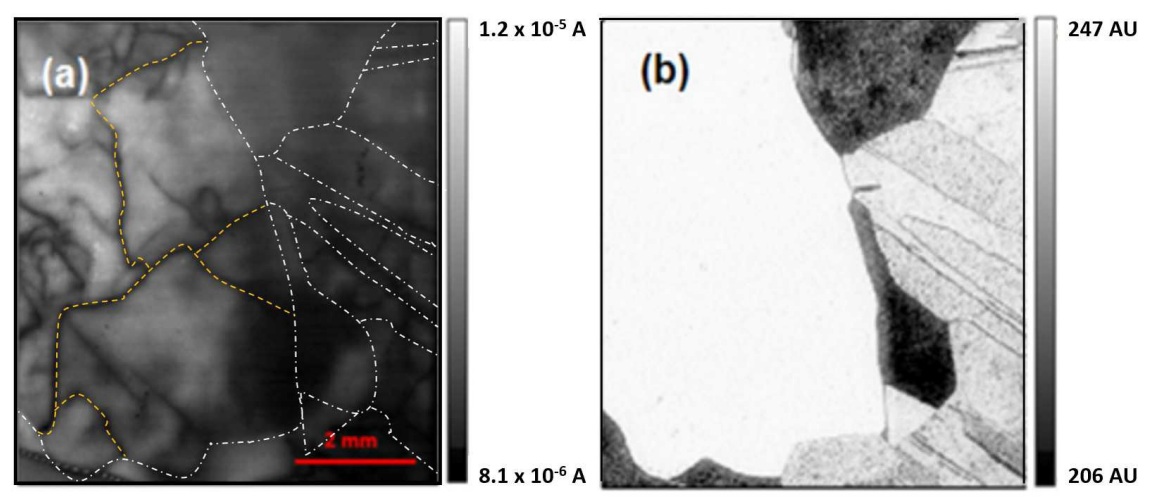

Fig. 1 LBIC image ( $853 \mathrm{~nm}$, x20 objective) (a), reflected light image (b), of a conventional cell. The dotted line (white) highlights the GBs, while the dotted line (yellow) highlights some electrically active subgrain boundaries revealed by LBIC but not in the reflected light image.

LBIC images of three sister cells are shown in Fig.2, one observes that the electrical activity of the defects is progressively reduced from bottom to top 
as the concentration of impurities is reduced due to segregation; a concomitant increase; from 16 to $17.8 \%$, of the cells efficiency is also observed. The recombination activity of the GBs is related to the density of dislocations in the boundary plane, which is associated with the type of boundary [12], and the impurity contamination [13].

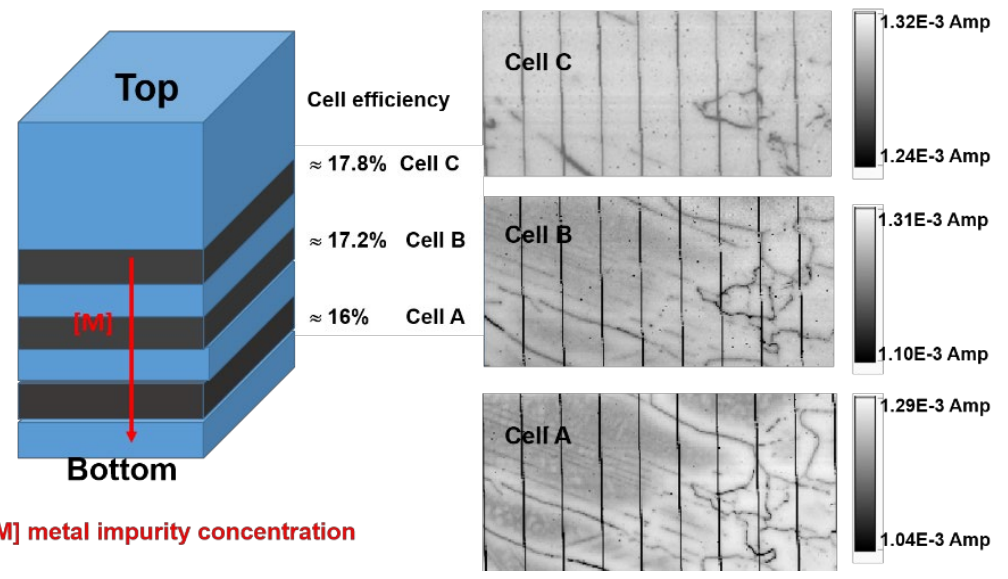

Fig. 2 Scheme of the UMG cells location along the ingot, and LBIC images of three sister cells from three regions of the ingot from the bottom to the middle (A, B and C), showing similar defect distribution, but a different level of electrical activity

The same defect structures were mapped by LBIC for the three samples, which permits a comparative analysis between them in order toevaluate the influence of impurity contamination. Cell A exhibits dark contrast for coincidence lattice site (CLS) GBs and random angle GBs (RAGBs). This contrast is substantially smoothed for cell B, especially for the GBs, albeit the main contrast features seen in cell A are still observed in cell B. Cell C presents some dark contrasted RAGBs, but most of the GBs and RAGBs revealed in cell A appear electrically inactive. The electrical activity of the defects is related to the impurity contamination and the structure of the defect itself. Transition metal impurities in this kind of material are usually present in concentration close to $1014-1016 \mathrm{~cm}^{-} 3$ [6], it decreases in the middle of the ingot, down to $\approx 1012-1013 \mathrm{~cm}^{-} 3$, with respect to the top and bottom zones, which are more contaminated. Cell C arises from the middle of the ingot, while cells $B$ and A are closer to the bottom, therefore, they contain a higher concentration of residual impurities. From these results it appears that the background impurity concentration plays a major role in the electrical activity of the defects. The activity of the GBs is related to the density of dislocations in the boundary plane, which the decoration with impurities makes them electrically active [12].

Cell A is the closest one to the ingot bottom, therefore, it is the one with the highest concentration of impurities. One observes around the high con- 
trasted dark lines (GBs and RAGBs) a bright contrasted halo, corresponding to an impurity denuded zone created by the gettering of impurities by the GBs. It is worth noting, that in the region presenting a higher concentration of boundaries, see the circle area in Fig.3, cell A, the gettering of impurities by the boundaries leaves a large area with bright LBIC contrast, corresponding to enhanced internal quantum efficiency (IQE). This is consistent with the strategy of decreasing the grain size with a structure of RAGBs (High performance multicrystalline Si- HPmc-Si), which results in enhanced cell efficiency [14]. The average LBIC current in this region is higher than the one measured in large grains, in spite of the carrier trapping by the boundaries. In the middle of large grains one observes inhomogeneous soft contrast forming a cellular like structure, associated with an inhomogeneous impurity distribution inside the grains, Fig.4a. This kind of distribution is not observed for all the grains, which suggests that it could be associated with the grain structure and orientation, resulting in the non homogeneous distribution of impurities. Therefore, dense arrays of RAGBs forming small grains should allow increasing the internal quantum efficiency (IQE) as compared to large grains, at least for highly contaminated material.
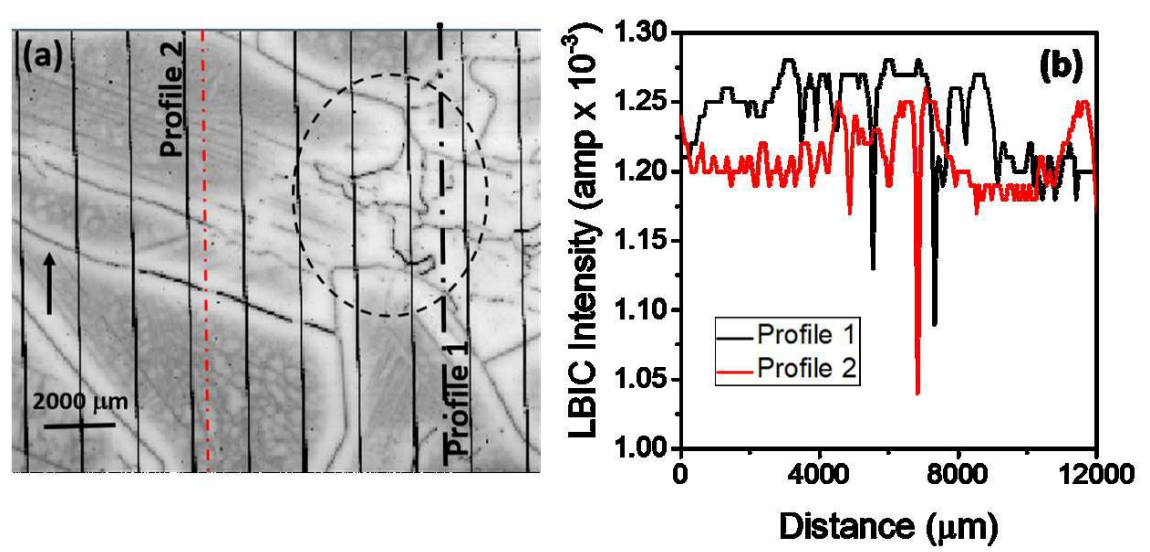

Fig. 3 The region inside the circle with a high concentration of RAGBs presents a higher LBIC signal than the large grains, due to the gettering of impurities by the RAGBs. The average LBIC intensity is enhanced in the region encircled in spite of the recombination activity of the RAGBs, see the LBIC profiles in the right panel (b), where a higher LBIC intensity is recorded along the profile 1 . Therefore, dense arrays of RAGBs forming small grains should allow increasing the internal quantum efficiency (IQE) as compared to large grains.

There are also dark boundaries around which the bright halo corresponding to the impurity depletion is less marked, see the arrow indicating a GB with a tenuous halo, Fig.3a. This suggests that the gettering of impurities is less efficient, even if some of them present dark contrast; therefore, the boundary structure itself may play a main role in impurity gettering. The largest bright halo, and therefore the most efficient impurity gettering is observed for RAGBs. Note that the LBIC profile around the boundaries does not only 


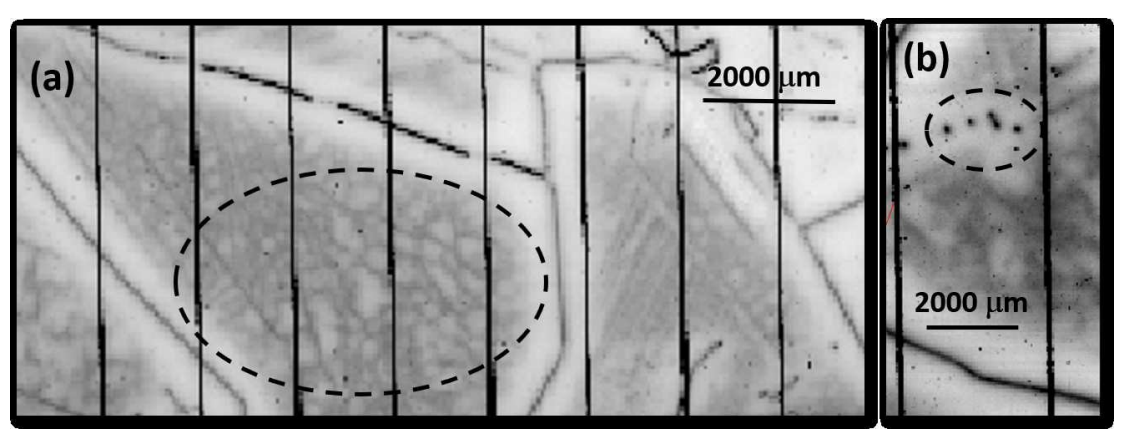

Fig. 4 a) LBIC cellular like distribution inside a large grain. b) LBIC dark spots surrounded by an impurity denuded halo (cell A).

reflect the capture of the minority carriers by the boundary but also it is correlated to the impurity distribution around the boundaries, which reduces the effective carrier lifetime and consequently the diffusion length, which is substantially shortened around the defects [15]. Generally, the contrast profile at both sides of the boundaries differs, which is related to the different distribution of impurities at both sides of the GB. One also observes dark contrasted spots inside the large grains, surrounded by a denuded halo. These dark spots seem to consist of dislocation clusters, which are very efficient in gettering impurities, Fig.4b. The dark contrast of the GBs is not homogeneous, but it presents different levels of contrast along the boundaries, which can be related to its ability to capture impurities.

Cell B is closer to the middle of the ingot, the contrast around the boundaries is greatly smoothed with respect to cell $A$, the GBs are much less visible in the LBIC images, Fig.2. Also, the impurity distribution inside the grains is different and the cellular like structure observed in cell $\mathrm{A}$ is anymore observed; furthermore, the denuded halo around the boundaries is significantly softened with respect to cell A.

Finally, in cell C most of the dark contrasted features have disappeared, concomitantly with the lower concentration of impurities in this part of the ingot, showing that the decoration with impurities plays a crucial role in the electrical activity of the crystal defects.

The LBIC contrasts profiles along equivalent lines for the three cells are shown in Fig.5. One observes a significant decrease of the LBIC contrast from cell A to cell $\mathrm{C}$. One also observes how the boundaries are slightly different in cells A and B, the grains have not walls parallel to the ingot axis, but they are tilted and subgrains are formed which change the defect structure along the ingot. The LBIC contrast of the three cells reveals that the passivation of the GBs can only be achieved below a certain threshold concentration of impurities. Cell $C$ is close to such threshold as many of the GBs are not electrically active; meanwhile, the passivation is not effective for the case of a high concentration of impurities, cell $\mathrm{A}$, and to a minor extent cell $\mathrm{B}$, for which most of the GBs are still electrically active 

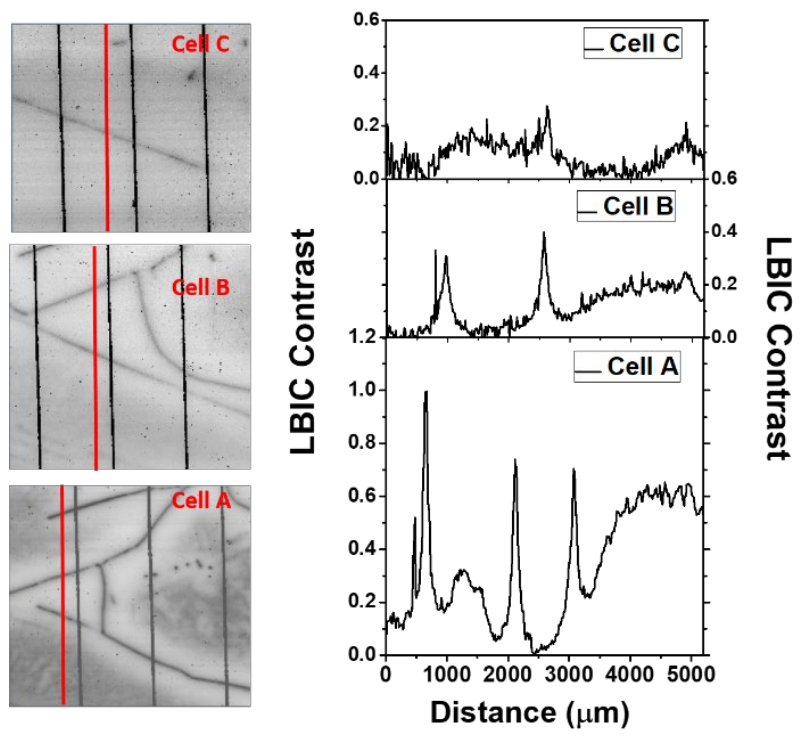

Fig. 5 LBIC contrast along the lines drawn in the LBIC images, showing the progressive reduction of the contrast from cell $\mathrm{A}$ to cell $\mathrm{C}$ as the electrical activity of the GBs decreases because of the lower concentration of impurities.

\section{Conclusions}

We have studied the electrical activity of UMG mc-Si solar cells by LBIC mapping. The use of sister cells permits a direct comparison of the electrical activity of the GBs at different levels of impurity contamination. The electrical activity of the GBs appears tightly related to the presence of impurities. RAGBs act as getter centers for impurities. Denuded zones around the GBs, especially the RAGBs, are observed in cells fabricated on wafers from the bottom of the ingot, which turn out to be highly contaminated. The LBIC contrast is progressively softened as one approaches the middle of the ingot where the impurity concentration is substantially reduced. The use of dense arrays of RAGBs permits to increase the IQE in this material.

Acknowledgments This work was supported by the project VA283P18 (Junta de Castilla y Leon) and project ENE2017-89561-C4-3-R (Spanish Ministry of Economics and Competitiveness), and European Fund for Regional Development (FEDER). 


\section{References}

1. B. Michl, M. Padilla, I. Geisemeyer, S. T. Haag, F. Schindler, M. C. Schubert, W. Warta; IEEE J. Photovolt. 4, 1502 (2014)

2. T. Trupke, R. A. Bardos, M. C. Schubert and W. Warta; Appl. Phys. Lett. 89, 044107 (2006)

3. O. Breitenstein, J. Bauer, T. Trupke, R. A. Bardos; Prog. in Photovoltaics: Res.and Appl. 16,325 (2008)

4. M. Padilla, B. Michl, B. Thaidigsmann, W. Warta, M.C. Schubert; Solar Energy Mater. and Solar Cells 120, 282 (2014)

5. T. Buonassisi, A. A. Istratov, M. D. Pickett, M. A. Marcus, T. F. Ciszek, E. R. Weber; APL 89, $042102(2006)$

6. S. Castellanos, K. E. Ekstrøm, A. Autruffe, M. A. Jensen, A. E. Morishige, J. Hofstetter P. Yen, B. Lai, G. Stokkan, C. del Cañizo, T. Buonassisi; IEEE J. Photovoltaics 6, 632 (2016)

7. K. Adamczyk, R. Søndena, G. Stokkan, E. Looney, M. Jensen, B. Lai, M. Rinio, M. Di Sabatino1 JAP 123, 055705 (2018)

8. P. Zheng, F. E. Rougieux, X. Zhang, J. Degoulange, R. Einhaus, P. Rivat, D. H. Macdonald; IEEE J. Photovolt. 7, 58 (2017)

9. J. Chen, T.Sekiguchi, S.Nara, D.Yang; J. Phys. 16, S211 (2004)

10. D.E.Apsnes, A.A. Studna; Phys. Rev. B 27, 985 (1983)

11. B. Moralejo, M. A. González, J. Jiménez, V. Parra, O. Martínez, J. Gutiérrez, O. Charro; J.Electron. Mater. 39, 663 (2010)

12. W. Seifert, G. Morgenstern, M. Kittler; Semicond. Sci. Technol. 8, 1687 (1993)

13. B. Chen, J. Chen, T. Sekiguchi, M. Saito, K. Kimoto; J.Appl. Phys. 97, 033701 (2005)

14. C.W. Lan, A. Lan, C.F. Yang, H.P. Hsu, M. Yang, A. Yu, B. Hsu, W.C. Hsu, A. Yang; J. Cryst. Growth 468, 17 (2017)

15. L.A. Sanchez, A. Moreton, M. Guada, S.Rodriguez-Conde, O.Martínez, J.Jiménez; MRS Adv. DOI: $10.1557 / \mathrm{adv} .2018 .366$ (2018) 